\title{
Magnetic Configuration and Modular Coil Design for the Chinese First Quasi-Axisymmetric Stellarator ${ }^{*)}$
}

\author{
Haifeng LIU ${ }^{1,4)}$, Akihiro SHIMIZU ${ }^{2)}$, Mitsutaka ISOBE ${ }^{2,3)}$, Shoichi OKAMURA ${ }^{2)}$, \\ Shin NISHIMURA ${ }^{2)}$, Chihiro SUZUKI ${ }^{2)}$, Yuhong XU ${ }^{1)}$, Xin ZHANG ${ }^{1)}$, Bing LIU $^{1)}$, \\ Jie HUANG $^{1)}$, Xianqu WANG ${ }^{1)}$, Hai LIU ${ }^{1)}$, Changjian TANG ${ }^{1,4)}$, Dapeng YIN $^{5)}$, \\ Yi WAN ${ }^{5)}$ and CFQS team ${ }^{1,2)}$ \\ ${ }^{1)}$ Institute of Fusion Science, School of Physical Science and Technology, Southwest Jiaotong University, \\ Chengdu 610031, China \\ ${ }^{2)}$ National Institute for Fusion Science, National Institutes of Natural Sciences, Toki 509-5292, Japan \\ ${ }^{3)}$ SOKENDAI (The Graduate University for Advanced Studies), Toki 509-5292, Japan \\ 4) Physics Department, Sichuan University, Chengdu 610041, China \\ ${ }^{5)}$ Hefei Keye Electro Physical Equipment Manufacturing Co., Ltd, Hefei 230000, China
}

(Received 28 December 2017 / Accepted 13 March 2018)

The Chinese First Quasi-axisymmetric Stellarator (CFQS) is a joint project of international collaboration. It is designed and fabricated by Southwest Jiaotong University (SWJTU) in China and National Institute for Fusion Science (NIFS) in Japan. In this work, a plasma boundary and modular-coil system for the CFQS have been designed and optimized via scan of the magnetic configurations with various aspect ratios. The present target parameters of the CFQS are as follows: toroidal periodic number, aspect ratio, magnetic field strength and major radius are 2, 4.0, 1.0 T, and $1.0 \mathrm{~m}$, respectively. The VMEC and NESCOIL codes are employed to obtain the optimum coil design via minimizing the normal component of the magnetic field on the target plasma boundary surface generated by the modular coils. In order to estimate the accuracy of the filament coils, the shape of the plasma boundary, rotational transform, depth of magnetic well and main Fourier components of magnetic field strength produced by the modular-coil system are compared with the target plasma boundary, rotational transform, depth of magnetic well and main Fourier components, correspondingly. This comparison between properties of the coil induced configuration and the target configuration shows a good agreement.

(c) 2018 The Japan Society of Plasma Science and Nuclear Fusion Research

Keywords: quasi-axisymmetric stellarator, modular coil, plasma boundary, configuration optimization

DOI: $10.1585 /$ prr.13.3405067

\section{Introduction}

On July 3rd, 2017, SWJTU and NIFS concluded the official agreement to design and operate collaboratively the CFQS based on the CHS-qa configuration, which indicates that the physics and engineering investigation on the advanced stellarator has been carried out in China. In recent years, helical systems (stellarators) have made a remarkable progress in magnetic fusion research. In 2015, Wendelstein 7-X at the Greifswald branch of Max Planck Institute for Plasma Physics achieved the first plasma [1], which is the world's largest fusion device of the stellarator type. In this year, the deuterium plasma experiment has been executed successfully on the Large Helical Device (LHD). One of the significant achievement is that ion temperature reaches the $10 \mathrm{keV}$ [2]. Advanced configuration design studies of helical systems have also been developed greatly, and a number of new configurations have

author'se-mail: hfliu@swjtu.edu.cn

*) This article is based on the presentation at the 26th International Toki Conference (ITC26). been proposed owing to the great freedom in the geometry of three-dimensional (3D) helical system structures such as quasi-symmetric configuration and quasi-isodynamic configuration. In such new configuration developments, the primary objectives were to improve neoclassical transport and explore high-beta configurations [3]. As for the quasi-symmetric configuration, there are three characteristic types of topologies, i.e. quasi-helical symmetry, quasiaxisymmetry and quasi-poloidal symmetry. The first experimental test of quasi-symmetry is the Helically Symmetric eXperiment (HSX) now operating at the University of Wisconsin [4].

Quasi-axisymmetric stellarators offer novel solutions for confining high- $\beta$ plasmas by combining best features of advanced tokamaks and optimized stellarators [5]. Using the 3D shaping freedom available in a stellarator, configurations can be designed that are MHD stable without nearby conducting structure, requiring no current drive at high $\beta$ and have good orbit confinement. The quasiaxisymmetry gives good orbit and/or neoclassical confine- 


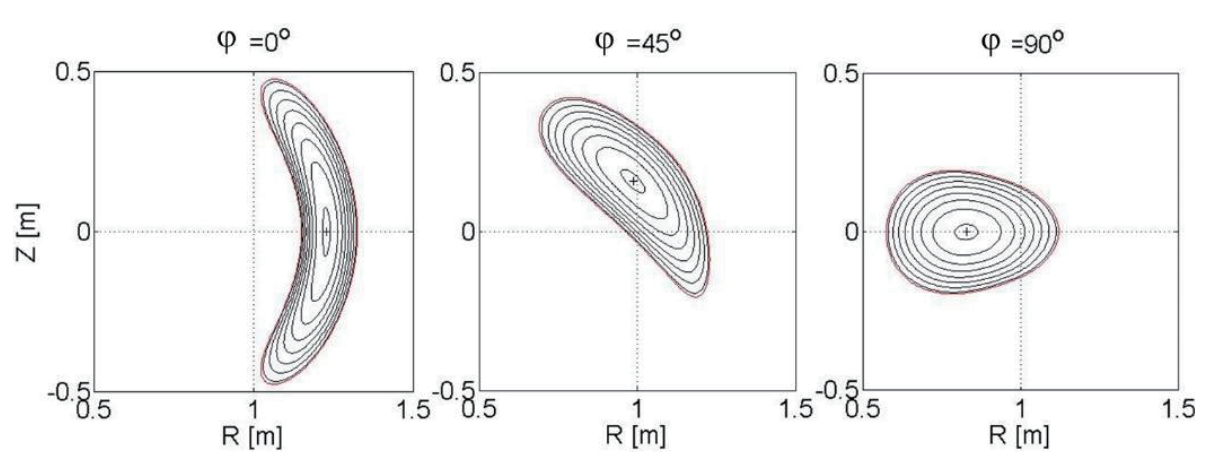

Fig. 1 Shapes of magnetic flux surfaces at the three different toroidal angles.

ment, equivalent to tokamaks. The reduced damping of toroidal rotations lead to improved confinement through the plasma sheared rotation [6]. Quasi-axisymmetric plasmas have a similar level of bootstrap current to tokamaks $[7,8]$. The increase of the rotational transform of high beta plasmas may contribute to better MHD properties and confinement. The rotational transform profile produced by the 3D shaping and bootstrap current can be designed to monotonically increase towards the plasma edge, like the core region of a 'reversed shear' advanced tokamak, which is utilized to stabilize neoclassical tearing modes, reduce equilibrium islands, and stabilize trapped-particle driven modes [9]. In addition, low aspect ratio achieved in the quasi-axisymmetric configuration is attractive in order to minimize the cost of near term experiments and the capital cost of possible future power plants. In this paper, we discuss the configuration characteristics and the design of the modular coil system for the CFQS.

\section{Configuration Characteristics}

The plasma boundary of the CFQS is originated from CHS-qa configuration by shrinking the shape of the plasma boundary of the CHS-qa [10]. Via the scan of major radius $(1.0 \mathrm{~m}-1.5 \mathrm{~m})$ and aspect ratio (3-5), the present candidate parameters of the CFQS configuration are token in account as follows: toroidal periodic number, aspect ratio, magnetic field strength and major radius are 2, 4.0, 1.0 T and $1.0 \mathrm{~m}$, respectively. The equilibrium was obtained with the VMEC code [11] fixed boundary calculation. The toroidal periodic number $N=2$ is selected, which guarantees to form the Tokamak-like configuration. A low aspect ratio is one of the important features of the CFQS design because of the advantage of compactness and economy, which could be used in future reactors. Additionally, nonaxisymmetric magnetic field components can be reduced due to its large toroidicity. From the core region to the edge, the vacuum rotational transform is designed between $2 / 6$ and 2/5 which is advantageous to avoid low-order rational surfaces. The presence of a magnetic well is capable to stabilize the MHD and reduce the island widths [12,13]. A standard expression for the plasma boundary is the Garabedian representation [14] which is described in cylindrical coordinates by Fourier decomposition, as follows:

$$
\begin{aligned}
& R(\theta, \zeta)=\sum\left(R_{\mathrm{mn}} \cos (m \theta-n N \zeta)\right) \\
& Z(\theta, \zeta)=\sum\left(Z_{\mathrm{mn}} \sin (m \theta-n N \zeta)\right) \\
& \varphi=\zeta
\end{aligned}
$$

where $\theta$ and $\zeta$ stands for mathematic/arbitrary poloidal and toroidal angels. The magnetic field strength of $3 \mathrm{D}$ equilibria can be specified conveniently in the Boozer coordinates and expanded in a formo of Fourier series:

$$
B\left(\psi, \theta_{\mathrm{B}}, \varphi_{\mathrm{B}}\right)=\sum\left(B_{\mathrm{mn}}(\psi) \cos \left(m \theta_{\mathrm{B}}-n N \varphi_{\mathrm{B}}\right)\right)
$$

The coefficients $B_{\mathrm{mn}}$ are refered to as the magnetic spectrum. The $N, \theta_{\mathrm{B}}$ and $\varphi_{\mathrm{B}}$ denote the toroidal periodicity, poloidal and toroidal angels in the Boozer coordinates. The quasi-axisymmetry means that only the first row of coefficients $B_{m, 0}$ are large enough to matter, as in tokamaks. The ordinary differential equations for guiding center orbits show that good neoclassical transport will prevail in the quasi-axisymmetric configuration. Figure 1 displays three poloidal cross-sections of the equilibrium magnetic surfaces for the plasma pressure-free case. These magnetic flux surfaces indicate large axisymmetric (or toroidal average) crescent, elongation and triangularity, which enhance stability of ballooning and kink modes.

Figure 2 (a) shows the rotational transform profile which displays the low magnetic shear exists in the CFQS configuration. The low magnetic shear is beneficial to the emergence of the internal transport barrier [15]. Figure 2 (b) shows the profile of magnetic well depth. It can be seen that the well structure is realized in the entire region which leads to the stability of MHD. Figure 2 (c) shows the contour of magnetic field strength at $\rho=6$ The dominant ripples are toroidal ones, indicating a strong axisymmetrylike congfiguration.

\section{Design of the Modular Coil System}

Vacuum equilibrium properties of a toroidal configuration are determined by the shape of the outmost closed flux surface (plasma boundary). Generally, considering the nested magnetic flux surfaces, the VMEC code enables to solve the three dimensional MHD equilibrium accurately 


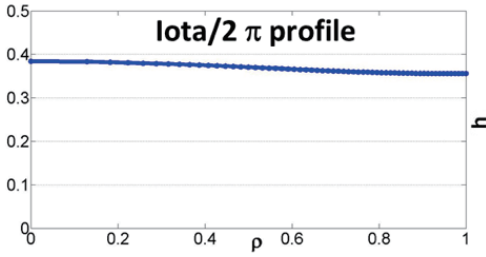

(a)

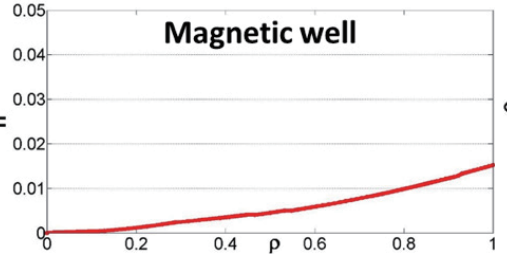

(b)

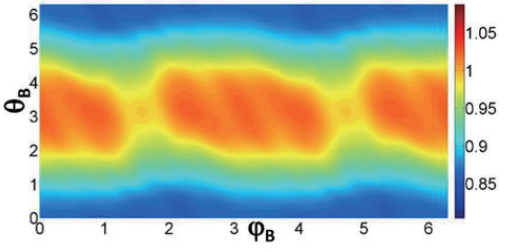

(c)

Fig. 2 Rotational transform profile, magnetic well depth profile and mod B contour at $\rho=6$.

Table 1 Parameters of three different types of coil systems.

\begin{tabular}{|c|c|c|c|c|c|c|c|c|}
\hline$A_{\mathrm{p}}$ & $R_{0}(\mathrm{~m})$ & $a(\mathrm{~m})$ & $\begin{array}{l}\text { Number } \\
\text { of coils }\end{array}$ & $\begin{array}{l}\text { Min. distance } \\
\text { between coils } \\
(\mathrm{cm})\end{array}$ & $\begin{array}{l}\text { Min. radius } \\
\text { of curvature } \\
(\mathrm{cm})\end{array}$ & $\mathrm{B} \cdot \mathrm{n} / \mathrm{B} \mid$ & $\begin{array}{l}\text { Current per } \\
\text { coil (MA) }\end{array}$ & $\begin{array}{l}\text { Cross section of } \\
\text { coils }\left(\mathrm{cm}^{2}\right)\end{array}$ \\
\hline 4.0 & 1 & 0.250 & 20 & 17.0 & 18.2 & $1.11 \%$ & 0.2500 & $17 \times 9$ \\
\hline 4.0 & 1 & 0.250 & 16 & 18.5 & 21.5 & $0.97 \%$ & 0.3125 & $18 \times 10$ \\
\hline 4.0 & 1 & 0.250 & 12 & 14.2 & 14.7 & $1.21 \%$ & 0.4167 & $19 \times 13$ \\
\hline
\end{tabular}

and efficiently. In order to achieve the target magnetic configuration, a modular coil system is necessary to be designed to reproduce the plasma boundary. Due to the Neumann boundary condition, the accuracy of the magnetic configuration induced by the coil system is dependent on the normal component of the magnetic field on the plasma boundary, which is expressed as $\mathbf{B} \cdot \mathbf{n}$ where $\mathbf{B}$ is the vacuum magnetic field generated from the coil system on the plasma boundary and $\mathbf{n}$ is the normal unit vector of this surface. Via the minimization of $\mathbf{B} \cdot \mathbf{n}$ on the plasma boundary, the modular coil geometry is optimized. Meanwhile, the engineering constraints are taken into account which are the minimum interval between adjacent coils and maximum curvature. They are under consideration to avoid the coil-coil overlap and reduce complexity of the coil shape. This optimization process is accomplished by the NESCOIL code [16]. In the design of the coil system for the CFQS, the coil numbers have been scanned to achieve an optimum modular coil system. The 20-coil, 16coil and 12-coil systems are designed respectively. The results of filament-coil optimization are listed in the Table 1.

In the Table 1 , the $A_{\mathrm{p}}, R_{0}$ and a denote respectively the aspect ratio, major radius and minor radius. In comparison of the physics and engineering constraints among them, the table indicates that the 16-coils system is advantageous, which shows that the minimum interval between adjacent filament coils is the widest; the minimum radius of curvature is the largest and the magnetic flux surface generated is the closest to the target surface. Figure 3 gives the of 16modular coil system. Due to the toroidal periodicity $=2$ and stellarator symmetry, the whole torus consists of four symmetric sections. Therefore, the coils system possesses
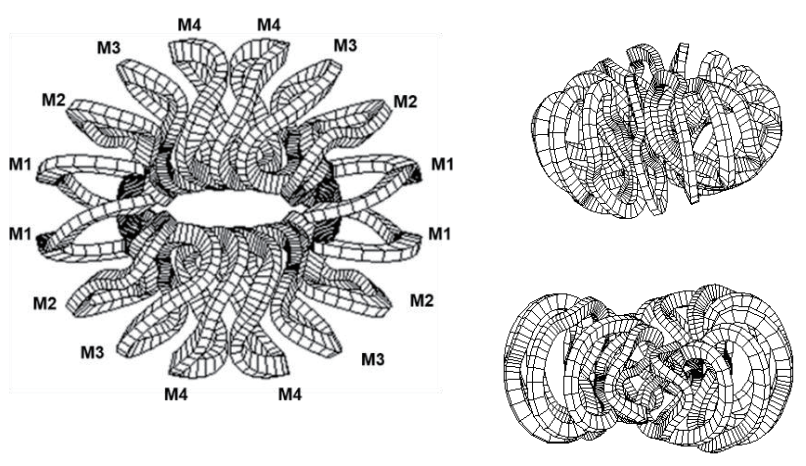

Fig. 3 Modular coils of the CFQS, the top view and side views at toroidal angle $=0^{\circ}$ (vertical elongation), and $90^{\circ}$ (horizontal elongation). The serial number of coils represents the various shapes of coils. The coil system comprises of four different shape coils.

four different shaped modular coils. The centerline of each finite sized coil is assigned by the corresponding filament coil. The all 16 filament coils are from the results of the NESCOIL code. The coil cross section is rectangular and the area is $18 \times 10 \mathrm{~cm}^{2}$ which includes copper conductor, insulation and coil casing.

In order to estimate the accuracy of the magnetic configuration induced by the 16-coil system, the coils generated magnetic flux surfaces, rotational transform and Fourier spectrum of the magnetic field strength are calculated. In the Fig. 4, Poincaré plots of magnetic flux surfaces and rotational transform induced by the modular coils are analyzed, assuming the coils are filament ones. At the toroidal angle $=0^{\circ}, 45^{\circ}$ and $90^{\circ}$, cross sections are 
displayed. The angle $=0^{\circ}$ and $90^{\circ}$ correspond to the vertically and horizontally elongated cross sections, respectively. The average of $\mathbf{B} \cdot \mathbf{n} /|\mathbf{B}|$ on the plasma boundary is below $1 \%$, which cannot be reduced from the viewpoint of the engineering. The rotational transform profile and magnetic well induced by coils and target profile are compared in the Fig. 4(d). Horizontal axis $\rho$ in this figure represents the normalized average minor radius. The figure shows a good agreement between coils induced rotational transform profile and depth of magnetic well and target ones. From the Figs. 4 (a) - (c), they depict a good coincidence in the shapes of a magnetic flux surface and that of target plasma boundary. It should be noted that the width of outmost flux surface produced by modular coils is larger than that of target plasma boundary, which is beneficial to raise the plasma volume by movable lim-

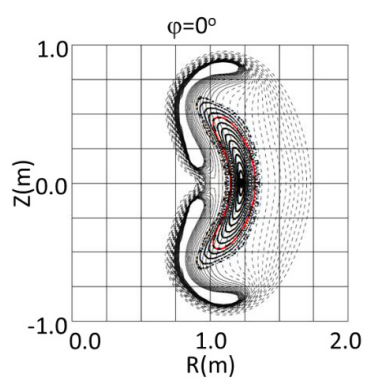

(a)

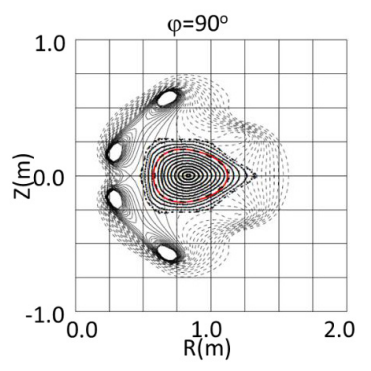

(b)

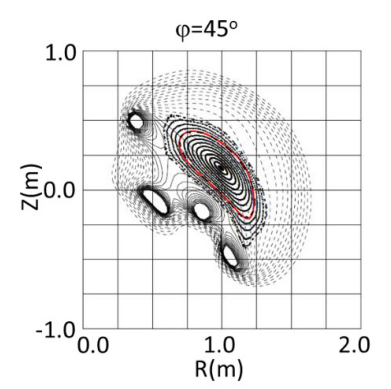

(b)

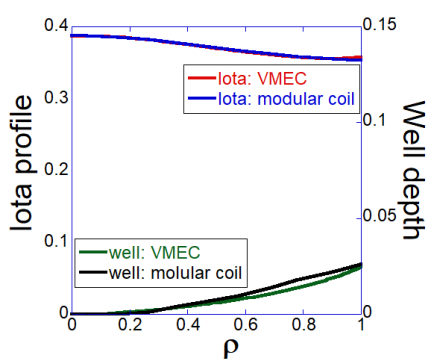

(d)
Fig. 4 Poincaré plots of magnetic flux surfaces at the toroidal angle $=0^{\circ}, 45^{\circ}$ and $90^{\circ}$ for (a) - (c) respectively. The red curve represents the target plasma boundary. (d) shows the comparison of the rotational transform and magnetic well between the configuration produced by modular coils and target one. iters. In order to precisely estimate the quasi-axisymmetry of the configuration, the magnetic field strength is decomposed into a Fourier spectrum in the Boozer coordinates, expressed as the Eq. (2). The Fig. 5 (a) depicts the spectrum of the magnetic field strength based on the the target magnetic configuration. To extinguish the small-amplitude components, the largest component $\mathrm{B}_{00}$ is omitted. In the Fig. 5 (a), the magnetic field strength is $1.0 \mathrm{~T}$. $\mathrm{B}_{10}$ is the dominant component resulting from the toroidicity. Others components, such as mirror ripple $\left(\mathrm{B}_{01}\right)$ and helical ripples $\left(\mathrm{B}_{11}, \mathrm{~B}_{12}\right)$, are much less than $\mathrm{B}_{01}$, which indicates a tokamak-like/quasi-axisymmetric configuration. In the Fig. 5 (b), coil induced spectrum of magnetic field strength is given. The amplitudes of main components of the target spectrum and the coil-induced spectrum are listed in the Table 2. The definition of the deviation rate is (target $B_{m n}{ }^{-}$ coil-induced $B_{m n}$ )/target $B_{m n}$. The deviation rate of $B_{01}$ is the largest. Other target components are very close to the coil-induced ones. On the basis of synthetical analyzation of Figs. 4, 5 and Table 1 the designed 16-coil system is well workable.

\section{Conclusion}

The CFQS design has been conducted as an international joint work between SWJTU and NIFS. The quasiaxisymmetric configuration is a tokamak-like configuration which can compatibly combine the good features of

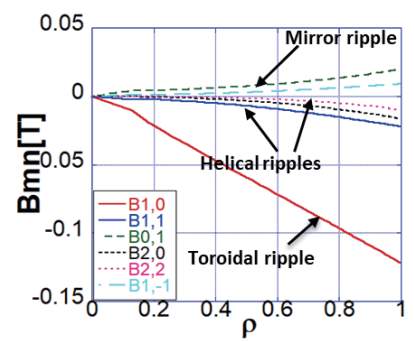

(a)

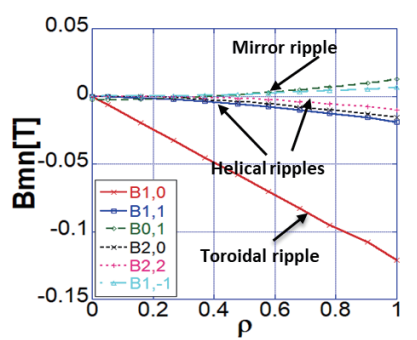

(b)
Fig. 5 Fourier spectrum of the magnetic field strength for the CFQS configuration in the Boozer coordinates. (a) $B_{m n}$ from VMEC results (target spectrum), (b) $B_{m n}$ generated by modular coils.

Table 2 Amplitudes of spectra.

\begin{tabular}{|l|l|l|l|l|l|l|}
\hline & $\mathrm{B}_{1,0}(\mathrm{~T})$ & $\mathrm{B}_{1,1}(\mathrm{~T})$ & $\mathrm{B}_{0,1}(\mathrm{~T})$ & $\mathrm{B}_{2,0}(\mathrm{~T})$ & $\mathrm{B}_{2,2}(\mathrm{~T})$ & $\mathrm{B}_{1,-1}(\mathrm{~T})$ \\
\hline Target component & -0.1209 & -0.0220 & 0.0198 & -0.0164 & -0.0100 & 0.0090 \\
\hline $\begin{array}{l}\text { Coil-induced } \\
\text { component }\end{array}$ & -0.1210 & -0.0189 & 0.0125 & -0.0158 & -0.0100 & 0.0066 \\
\hline Deviation rate & $0.83 \%$ & $14.09 \%$ & $36.87 \%$ & $3.66 \%$ & $0.00 \%$ & $26.67 \%$ \\
\hline
\end{tabular}


advanced tokamaks and stellarators, such as no requirement of the inductive plasma current, the steady-state operation, the reduced neoclassical transport, etc. In this paper, the modular coil system for the CFQS is designed successfully to achieve the quasi-axisymmetry. The present candidate parameters are that the toroidal periodic number $=2$, aspect ratio $=4.0$, magnetic field strength $=1.0 \mathrm{~T}$ and major radius $=1.0 \mathrm{~m}$. There are two steps to obtain the coil system which is able to realize the target magnetic configuration and can be fabricated from the engineering aspect. 1) considering the physics constraint, $\mathbf{B} \cdot \mathbf{n} /|\mathbf{B}|$ at the plasma boundary, and the fabrication constraints including the radius of coil curvature and distance between adjacent coils, the discrete coils are generated by NESCOIL code. 2) via the comparison between properties of the coils induced magnetic configuration and target configuration, the accuracy of the coil system is evaluated to guarantee the target configuration can be reproduced by the designed coil system.

\section{Acknowledgments}

We gratefully acknowledge the strong support and encouragement from H. Takeiri at NIFS and W.G. Zhang at SWJTU. This work was partly supported by the National Natural Science Foundation of China under Grant Nos. 11605145 and 11647314, the China Postdoctoral Science Foundation under Grant No.2016M600740, the Fundamental Research Fund for the Central Universities under Grant No. 2682016CX060 and NIFS General Collabora- tion Project budget under Grant No. NIFS17KBAP034.

[1] D. Clery, "The Bizarre Reactor That Might Save Nuclear Fusion" Science Magazine, 2015.

[2] M. Osakabe, Y. Takeiri, T. Morisaki et al., Fusion Sci. Technol. 72, 199 (2017).

[3] S. Okamura, K. Matsuoka, S. Nishimura et al., Nucl. Fusion 41, 1865 (2001).

[4] A.F. Almagri, D.T. Anderson, F.S.B. Anderson et al., IEEE Trans. Plasma Sci. 27, 114 (1999).

[5] S. Okamura, K. Matsuoka, S. Nishimura et al., Nucl. Fusion 44, 575 (2004).

[6] M. Isobe, S. Okamura, K. Matsuoka et al., Annual Report of National Institute for Fusion Science, 2001: 320.

[7] M. Isobe, S. Okamura et al., J. Plasma Fusion Res. SERIES 5, 360 (2001).

[8] M. Isobe et al., 28th EPS conference on Contr. Fusion and Plasma Phys. Funchal, 18-22 Jun 2001, 25A, 761 (2001).

[9] M.C. Zarnstorff, L.A. Berry, A. Brooks et al., Plasma Phys. Control. Fusion 43, A237 (2001).

[10] A. Shimizu, S. Okamura, M. Isobe et al., Fusion Eng. Des. 65, 109 (2003).

[11] S.P. Hirshman et al., Comput. Phys. Commun. 43, 143 (1986).

[12] J.R. Cary and M. Kotschenreuther, Phys. Fluids 28, 1392 (1985).

[13] Y. Xu, Matter and Radiation at Extremes 1, 4, 192 (2016).

[14] F. Bauer, O. Betancourt and P. Garabedian, Magnetohydrodynamic Equilibrium and Stability of Stellarators (Springer, 1984).

[15] Y. Kishimoto et al., Plasma Phys. Control. Fusion 40, A663 (1998).

[16] M. Drevlak, Fusion Technol. 33, 106 (1998). 